\title{
Temsirolimus in the treatment of relapsed and/or refractory mantle cell lymphoma
}

\author{
This article was published in the following Dove Press journal: \\ Cancer Management and Research \\ 25 June 2010 \\ Number of times this article has been viewed
}

\section{S Galimberti \\ $M$ Petrini}

Department of Oncology, Transplant and Advances in Medicine, Section of Hematology, University of Pisa, Pisa, Italy
Correspondence: Sara Galimberti Department of Oncology, Transplant and Advances in Medicine, Section of Hematology, University of Pisa, Ospedale S Chiara-Via Roma, 67-56I26 Pisa, Italy Tel +39050992815

Fax +39050830I62

Email s.galimberti@med.unipi.it

\begin{abstract}
Patients with mantle cell lymphoma (MCL) have a poor prognosis; consequently, new therapeutic approaches, such as rapamycin and its derivates, mammalian target of rapamycin (mTOR) inhibitors, are warranted. Temsirolimus (also known as CCI-779), a dihydroester of rapamycin, in MCL cell lines inhibited mTOR, downregulated p21 and v-Raf, and induced autophagy.The first clinical trial in MCL patients was performed using $250 \mathrm{mg}$ of temsirolimus weekly for 6-12 cycles. The overall response rate was $38 \%$; the median time to progression was 6.5 months, median overall survival was 12 months, and the median duration of response was 6.9 months. At lower dose (25 mg/week), the overall response rate was $41 \%$, median overall survival was 14 months, and time to progression was 6 months. In another trial, 162 patients were randomly assigned to receive temsirolimus at 2 different doses (175 mg/week for 3 weeks, then $75 \mathrm{mg}$ or $25 \mathrm{mg} /$ week) or a treatment chosen by the investigator among the most frequently adopted single agents for treatment of relapsed MCL. Patients treated with 175/75 mg of temsirolimus had significantly higher response rates and longer progression-free survival than those treated with investigator's choice therapy. These data support the use of mTOR inhibitors for the treatment of MCL, probably in combination with other agents, such as antiangiogenic drugs or histone acetylase inhibitors.
\end{abstract}

Keyword: mTOR rapamycin, PI3K/Akt, p7056K, 4E-BP1

\section{Mantle cell lymphoma}

Mantle cell lymphoma (MCL), which accounts for approximately $6 \%$ of all nonHodgkin lymphomas, represents the subtype where mammalian target of rapamycin (mTOR) inhibitors appear as more promising compounds in its treatment. The interest in application of these drugs to treat MCL has grown as it has been demonstrated that cyclin D1, overexpressed in this histotype of lymphoma, is an important downstream target of the mTOR signaling pathway.

Nevertheless, it has been reported that mTOR inhibitors inactivate other genes associated with the pathogenesis of MCL, such as vascular endothelial growth factor (VEGF), Ras, Raf, extracellular regulated kinase (ERK), and nuclear factor-kappa B. Moreover, the interest in this class of compounds has increased as more effective therapeutic strategies are required for MCL treatment, which has been demonstrated by the low response and survival rates observed in MCL patients treated with this drug.

Indeed, notwithstanding the most recent and effective therapeutic approaches adopted, the clinical evolution of most patients with MCL is still aggressive, with median overall survival not exceeding 3-4 years. ${ }^{1}$ Given the poor complete remission rates achieved with conventional regimens, chemotherapy combinations have been 
investigated to improve the outcomes: one of these regimens is the hyper-CVAD (fractionated cyclophosphamide, vincristine, doxorubicin, dexamethasone, alternating with high-doses methotrexate and cytarabine). This regimen, associated with the anti-CD20 monoclonal antibody rituximab, has shown good efficacy and tolerability in MCL, offering high response rates and 3-year failure-free survival and overall survival of $64 \%$ and $82 \%$, respectively. ${ }^{2}$ Analogously, rituximab combined with cyclophosphamide, adriamycin, vincristine and prednisone (R-CHOP) has been shown to be significantly superior to $\mathrm{CHOP}$ in overall response rate $(94 \%$ vs $75 \%$ ) and time to treatment failure (21 vs 14 months), with acceptable toxicity. ${ }^{3}$ In conclusion, the responses to second-line treatments of about $30 \%$ of patients affected by MCL relapse are still not fully satisfactory: rituximab associated with fludarabine, cyclophosphamide, and mitoxantrone (R-FCM) offered only 29\% of complete remission; ${ }^{4}$ radioimmunotherapy resulted in $30 \%$ of complete responses, with median event-free survival of 6 months and median overall survival of 21 months. ${ }^{5}$ Moreover, an objective response was achieved in $45 \%$ of patients receiving bortezomib, with median progression-free survival of only 6 months. ${ }^{6}$ Fiftythree percent of patients responded well to lenalidomide, with a median duration of response of 13.7 months and median progression-free survival of 5.6 months. ${ }^{7,8}$

\section{Targeting mTOR pathway}

In the mid-1970s, a strain of Streptomyces hygroscopicus, producing a compound with potent antifungal activity, was isolated from the soil samples collected from the Rapa Nui Island in the South Pacific. ${ }^{9}$ From the geographical origin, the purified antibiotic was named "rapamycin." antifungal activity, rapamycin was found to inhibit immune responses in mammals by depressing lymphocyte proliferation and to exert a cytostatic activity in several in vitro and in vivo cancer models. ${ }^{11}$ In the 1990 s, rapamycin was found to exert its activities by an evolutionarily conserved pathway that integrates signals from growth factors, nutrients, and energy status. The activation of mTOR pathway signifies a decision point that takes into account the availability of materials required for cell growth (amino acids, glucose, and energy) and the growth-regulating signals (hormones and growth factors). Thus, the cells are protected from outside signals to grow and still proliferate when nutrients and energy inside are not sufficient (Figure 1). ${ }^{12}$

The human MTOR gene (GeneID: 2475) maps to chromosome 1p36.2 and encodes a 289-kDa protein containing a kinase catalytic domain, 20 HEAT (Huntington elongation

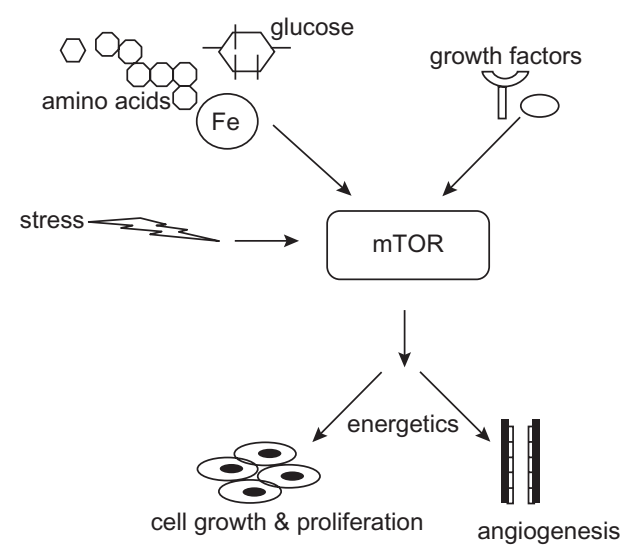

Figure I mTOR as central regulator of the cell nutrition and growth. Conditions outside of the cell, such as nutrient and energy levels, growth factors, hormones, and stressful conditions, control the mTOR activation. After activation, mTOR stimulates cell growth, increases angiogenesis, and responds to bioenergetics necessities.

factor 1A-protein phosphatase) repeats, an autoinhibitory repressor domain, and the FRB domain, responsible for interaction with FKBP12, a cofactor and transporter for rapamycin. ${ }^{13,14}$

The mTOR protein participates in 2 signaling complexes: mTORC1 and mTORC2. mTORC1, which is sensitive to rapamycin, includes mTOR, Raptor (regulatory-associatedprotein of mTOR), and G $\beta \mathrm{L}$ (G protein $\beta$-subunit-like protein). This complex reacts with signals that originate from growth factors, energy status, nutrient availability, hypoxia, reactive oxygen species, deoxyribonucleic acid (DNA) damage, and osmotic condition of environment. ${ }^{15}$ mTORC1 is activated by numerous upstream signalling pathways, including PI3K/Akt, Ras/MAPK, and Bcr-Abl1, whereas the negative regulators are phosphatase and tensin homolog (PTEN), LKB1, and the heterodimer TSC1 (tuberous sclerosis complex 1 or amartin)/TSC2 (tuberous sclerosis complex 2 or tuberin) ${ }^{16}$ (Figure 2). Activated Akt ${ }^{17}$ and RasRaf-ERK pathways phosphorylate TSC1/TSC2, leaving the Rheb protein GTP-bound and capable of interacting with and activating mTOR. ${ }^{18}$

The activity of PI3K is countered by PTEN; loss of PTEN has been reported in several solid tumors and lymphomas and seems to correlate with sensitivity to rapamycin derivatives. ${ }^{19}$

mTORC2 complex, rapamycin insensitive, contains mTOR, GßL, Rictor (rapamycin-independent companion of mTOR), and mSIN1 (mammalian stress-activated protein kinase interacting protein 1). ${ }^{20}$ It is believed that various growth factors contribute to mTORC2 activation. This complex regulates cytoskeleton organization and activates Akt, thus representing a further important level of self-regulation of the mTOR pathway. ${ }^{21}$ 


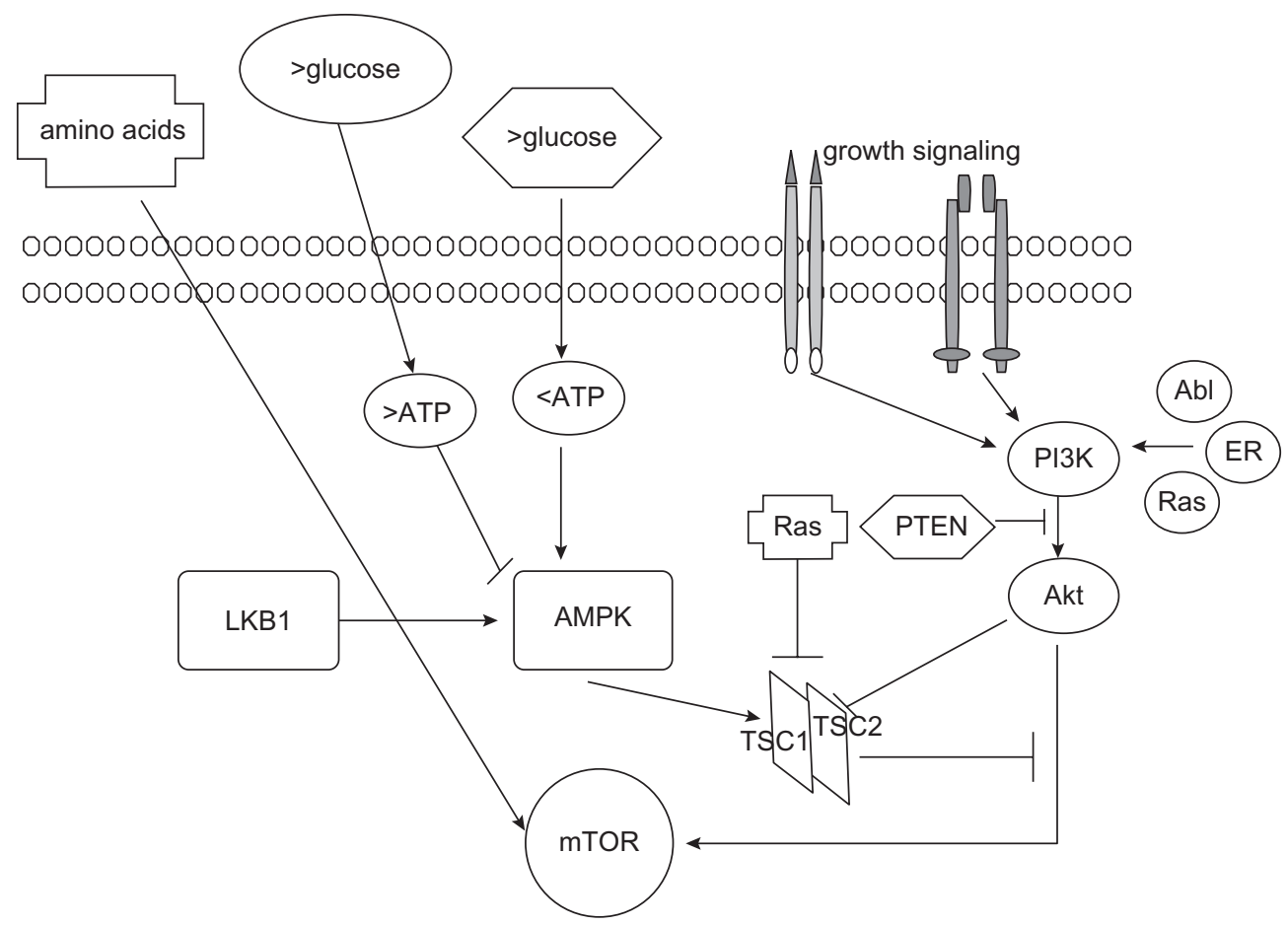

Figure 2 mTOR upstream pathway. Amino acid, glucose levels, growth factors, and genes, such as Bcr-Abl and Ras, induce activation of the PI3K/Akt pathway and consequently of the mTOR. The Akt pathway is inhibited by PTEN and LKBI through the TSCI-TSC2 complex.

Upon activation, mTOR facilitates cell cycle progression from $G_{1}$ to $S$ phase by phosphorylation of p70S6 kinase (p70S6K) and 4E-binding protein 1 (4E-BP1). p70S6K phosphorylates and activates $\mathrm{S} 6$, a ribosomal subunit involved in initiating translation of $5^{\prime}$ terminal oligopyrimidine tract-containing mRNA encoding components of the protein synthesis machinery. The mTOR-mediated phosphorylation of 4E-BP1 diminishes the stability of the 4E-BP1/eIF4E complex and facilitates the eIF4E action, enhancing translation of several mRNAs, such as cyclin D1, c-myc, hypoxia-inducible factor $1 \alpha(\mathrm{HIF} 1 \alpha)$, ornithine decarboxylase, VEGF, fibroblast growth factor, and ribosomal proteins (Figure 3).22,23

\section{mTOR inhibitors in hematological malignancies}

Rapamycin (sirolimus) was the first mTOR inhibitor used in clinical practice $;{ }^{24}$ more recently, several rapamycin analogs have been tested in clinical trials for solid and hematological malignancies: temsirolimus (CCI-779; Wyeth Pharmaceuticals, Madison, New Jersey, USA), everolimus (RAD001; Novartis, Basel, Switzerland), and deforolimus (MK-8669, AP23573; Merck and Ariad Pharmaceuticals, Cambridge, Massachusetts, USA).

Because mTOR is important for cell proliferation in several hematological malignancies, mTOR inhibitors have been used either as single drugs or in combination with conventional chemotherapeutic agents or monoclonal antibodies in acute/ chronic lymphocytic or myeloid leukemia, multiple myeloma, myelodysplastic syndromes, and lymphomas. mTOR inhibitors have been shown to be also effective against acute lymphoblastic leukemia, even when combined with methotrexate, anthracyclines, etoposide, or corticosteroids. ${ }^{24-27}$

Rapamycin restored sensitivity to steroid-resistant cells ${ }^{28}$ and to tyrosine kinase inhibitors in resistant patients with

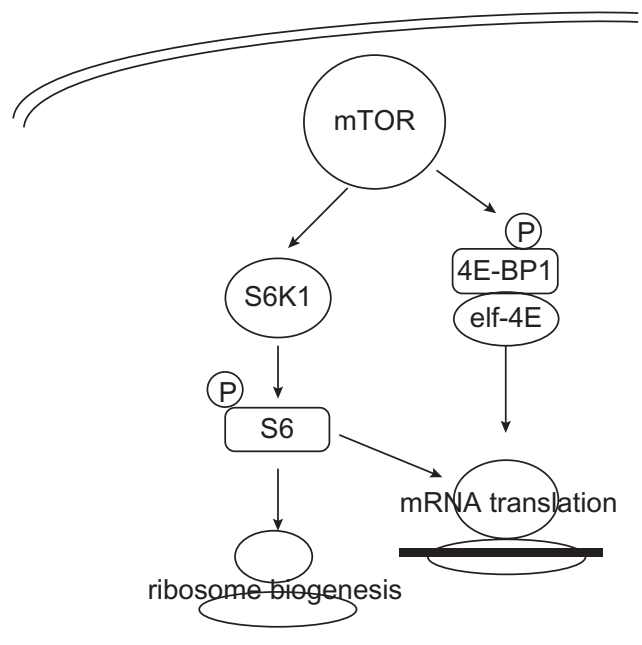

Figure 3 mTOR downstream pathway. mTOR activates the kinase activity of S6KI and mediates the release of elF4E translation initiating factor, thus regulating the synthesis of proteins involved in growth, proliferation, survival, angiogenesis, and bioenergetics. 
chronic myeloid leukemia. ${ }^{29}$ In acute myeloid leukemia, where Akt is activated, phosphorylation of p70S6K and 4EBP-1 is significantly inhibited by everolimus, especially when combined with Ara-C. ${ }^{30}$ Also in myelodysplastic syndromes, activation of the Akt/mTOR pathway in high-risk patients was described. ${ }^{31}$

The PI3K/Akt pathway is frequently activated in multiple myeloma, where mTOR inhibitors were effective. ${ }^{32,33}$ In a phase II trial, 16 patients with relapsed refractory multiple myeloma received $25 \mathrm{mg}$ of intravenous temsirolimus weekly until progression, with overall response rate of $38 \%$ and median time to progression of 138 days. ${ }^{34}$

In Hodgkin's lymphoma, everolimus downregulated the truncated isoform of the transcription factor CCAAT enhancer binding protein beta, which inhibits NF-kB activity. ${ }^{35}$ Rapamycin has been shown to inhibit mTORC1 also in diffuse large B-cell lymphoma cell lines ${ }^{36}$ and to overcome the P-glycoprotein-mediated multidrug resistance in B-lymphoma cell lines. ${ }^{37}$ In primary effusion lymphoma, rapamycin reduced the accumulation of ascites and extended mouse survival, with significant reduction in levels of circulating VEGF. ${ }^{38}$

Moreover, rapamycin showed a strong antiproliferative effect on B-cell lines derived from organ transplant recipients with Epstein-Barr virus-associated post-transplant lymphoproliferative disorders. ${ }^{39}$

mTOR inhibitors were effective in follicular lymphoma, where neoplastic cells display phosphorylation of p70S6K and 4E-BP1. ${ }^{40,41}$

In chronic lymphocytic leukemia (CLL) cells, where PI3K is constitutively active, ${ }^{42}$ cycle arrest induced by rapamycin was accompanied by reduced expression of cyclins D3, A, and E. ${ }^{43}$ A phase II trial with oral everolimus was conducted in 7 patients with CLL relapsed after at least 2 lines of therapy. Disease stabilization was observed in 3 patients and partial response in 1 patient; however, the trial was precociously stopped because of infectious toxicity. ${ }^{44}$

\section{MCL and $m$ TOR inhibitors}

Overexpression of the cyclin D1, product of the oncogene $\mathrm{Bcl}-1$, is considered the primary genetic event in MCL and hence mTOR inhibitors are used in the treatment of MCL. Cyclin D1 plays a critical role in cell transition from the $G$ to $S$ phase in response to mitogens. ${ }^{45}$ The overexpression of cyclin $\mathrm{D} 1$ is due to the translocation $\mathrm{t}(11 ; 14)(\mathrm{q} 13 ; \mathrm{q} 32)$ juxtaposing the Bcl-1 (CCND1) gene on chromosome 11 to the immunoglobulin heavy chain locus (IgVH) on chromosome $14 .{ }^{46} \mathrm{t}(11 ; 14)$ (q13;q32) is identified in $50 \%-70 \%$ of MCL cases based on the type of the method used.

Cyclin D1, in association with cyclin-dependent kinase-4 and -6 , induces the cell to enter the $\mathrm{S}$ phase by phosphorylating the retinoblastoma tumor-suppressing protein, which binds to transcription factors, including E2F. ${ }^{47}$

In addition to the overexpression of cyclin D1, other genes that overexpress in MCL, such as VEGF and Raf-1, represent further possible targets for mTOR inhibitors.

Indeed, a constitutive activation of Akt and mTOR pathways either in MCL cell lines (Granta 519, Jeko-1, and SP-53) or in primary cultures from $30 \%$ of MCL patients has been reported. ${ }^{48,49}$ Moreover, mTOR inhibitors in MCL could synergize with other "canonical" agents, such as vincristine, doxorubicin, bortezomib, and rituximab, with resulting inhibition of Raf-1, MAPK, and mTOR. ${ }^{50,51}$

Nevertheless, the data on the in vitro activity of different mTOR inhibitors in MCL cell lines are conflicting; ${ }^{52}$ all compounds induced cell cycle arrest in $\mathrm{G}_{0} / \mathrm{G}_{1}$ phase, temsirolimus reduced p21 expression, and rapamycin increased p27 expression; however, the data on cyclin D1 levels are still not clear (Table 1).

In several MCL cell lines, pharmacological inhibition of the PI3K/Akt pathway by rapamycin was associated with downregulation of cyclin D1 and the antiapoptotic proteins cFLIP, Bcl-X $\mathrm{L}_{\mathrm{L}}$, and Mcl-1. ${ }^{53}$ Treatment with rapamycin inhibited the proliferation of Granta and NCEB cells, with accumulation of cells in $G_{1}$ phase, without modification of the apoptotic process. The expression of cyclins D3, E, and A was strongly reduced in both cell lines, while cyclin D1 expression was not changed. ${ }^{54,55}$

In another in vitro study, rapamycin inhibited cell proliferation and induced cell cycle arrest in $\mathrm{G}_{0} / \mathrm{G}_{1}$ phase mediated by cyclin D3 and p27 deregulation. In the SP-53 cell line

Table I In vitro activity of mTOR inhibitors on MCL cell lines: relevant data from literature

\begin{tabular}{|c|c|c|c|c|c|c|}
\hline$\overline{\text { Drug }}$ & Antiproliferative effect & p27 & Akt & Cyclin DI & Cyclin D3 & Reference \\
\hline Rapamycin & $\mathrm{G}_{0} / \mathrm{G}_{1}$ arrest & Increased & No change decreased & No change & Decreased & Dal Col et $\mathrm{al}^{48}$ \\
\hline Rapamycin & $\mathrm{G}_{0} / \mathrm{G}_{1}$ arrest & & & Decreased & & Peponi ${ }^{53}$ \\
\hline Rapamycin & $\mathrm{G}_{0} / \mathrm{G}_{1}$ arrest & No change & & No change & Decreased & Hipp et $\mathrm{al}^{54}$ \\
\hline Temsirolimus & & & Decreased & & & Younes $^{52}$ \\
\hline Temsirolimus & $\mathrm{G}_{0} / \mathrm{G}_{\mathrm{l}}$ arrest autophagy & No change & Increased & No change & No change & Yazbeck et al ${ }^{57}$ \\
\hline Everolimus & $\mathrm{G}_{0} / \mathrm{G}_{1}$ arrest & & & & & Haritunians et a $\left.\right|^{50}$ \\
\hline
\end{tabular}


only, rapamycin downregulated cyclin D1 levels; this effect on cyclin D1 was observed only in cell lines where GSK3 $\beta$ was activated. ${ }^{48}$

In MCL, GSK3 $\beta$ is inactivated in about half the cases as a consequence of $\mathrm{Wnt}$ stimulation. After that, $\beta$-catenin is released and translocated into the nucleus, where it upregulates the transcription of several genes, including cyclin D1. ${ }^{56}$

In MCL cell lines, cyclin D1 was unmodified by temsirolimus, ${ }^{57}$ which downregulated p21, thus inducing a cell cycle block in the $\mathrm{G}_{1}$ phase and exerting a cytostatic rather than a cytotoxic action.

Interestingly, temsirolimus showed a synergistic antineoplastic activity with vorinostat, probably adding to the proapoptotic effect exerted by the histone acetylase inhibitors, the induction of autophagy. Autophagy is a reversible, bidirectional process; it not only can enhance and promote survival under stressful conditions but also can lead to cell death. In an in vitro study on MCL cell lines, temsirolimus inhibited mTOR, downregulated p21, and induced autophagy without any effect on pERK, Bcl-2, Bax, Bad, and beclin-1. ${ }^{52}$

Another factor that supports the use of mTOR inhibitors in MCL is the inhibition of angiogenesis. Indeed, TORC1 activation results in upregulation of $\mathrm{HIF} 1 \alpha$ that leads to increased expression of angiogenic factors, such as VEGF and platelet-derived growth factor- $\beta^{58}$ In well-oxygenated cells, HIF $1 \alpha$ is continuously produced and degraded by proteosomes. In hypoxic cells, HIF $1 \alpha$ translocates to the nucleus, initiating the transcription of genes involved in glycolysis, angiogenesis, cell survival, and metastasis. As VEGF is one of the regulators of the PI3K/Akt activity, increased levels of VEGF induce a further activation of mTOR. Inhibition of mTOR activity affects angiogenesis by reducing the expression of HIF $1 \alpha$ and by inhibiting the ability of cells to respond to the mitogenic effect of VEGF.

High levels of VEGF have been associated with poor outcomes in acute lymphoblastic leukemia ${ }^{59}$ and nonHodgkin lymphomas. ${ }^{60,61}$ In MCL, VEGF expression has been reported in $41 \%$ of cases, with an evident negative prognostic significance. ${ }^{62}$

Recently, our group showed that 2 VEGF polymorphisms associated with higher plasmatic protein levels, $\mathrm{G}+405 \mathrm{C}$ and $\mathrm{C}+936 \mathrm{~T}$, were more frequently detected in MCL cases than in healthy controls, thus supporting the hypothesis that some VEGF genotypes would increase the risk of development of this lymphoma. ${ }^{63}$

Another upstream regulator of mTOR, particularly interesting in MCL, is the Ras/Raf/ERK/MAPK pathway. ${ }^{64} \mathrm{~V}$-Raf has been reported to act synergistically with c-Myc to induce B-cell tumors in a murine model ${ }^{65}$ In a series of patients with MCL and receiving R-hyper-CVAD, our group previously reported that c-Myc overexpression was associated with shorter overall and progression-free survival. ${ }^{66}$ Like c-Myc, Mcl-1 is overexpressed in MCL; ${ }^{67}$ this gene, a member of the bcl-2 family, is translationally regulated by mTORC1 and is involved in the antiapoptotic action of mTOR. ${ }^{68}$

Syk, a tyrosine kinase involved in B-cell receptor signalling, has been reported to be amplified at the DNA level and overexpressed at both RNA and protein levels in MCL cell lines and in a small subset of clinical samples. As inhibition of Syk resulted in potent inhibition of mTOR activity in follicular cells, mantle cells, Burkitt, and diffuse large B-cell lymphoma cell lines, this tyrosine kinase would represent a further good target for mTOR inhibitors. ${ }^{69}$

\section{Temsirolimus for relapsed MCL}

Temsirolimus (also known as CCI-779), a dihydroester of rapamycin suitable for intravenous use, is currently under trial for use in solid tumors, such as renal cancer, ${ }^{70}$ breast cancer, ${ }^{71}$ sarcomas, ${ }^{72}$ and prostate cancer. ${ }^{73}$ In hematology, it has been tested in MCL, multiple myeloma, and acute myeloid leukemia. ${ }^{74}$

The prototype mTOR inhibitor, oral rapamycin, is poorly soluble and undergoes extensive first-pass metabolism, leading to low and potentially variable absorption and exposure.

Table 2 Temsirolimus in MCL patients: results from clinical trials

\begin{tabular}{|c|c|c|c|c|c|c|}
\hline Schedule & $\begin{array}{l}\text { No. of } \\
\text { patients }\end{array}$ & $\begin{array}{l}\text { Overall } \\
\text { response }\end{array}$ & $\begin{array}{l}\text { Complete } \\
\text { response }\end{array}$ & $\begin{array}{l}\text { Median overall } \\
\text { survival }\end{array}$ & $\begin{array}{l}\text { Median } \\
\text { progression- } \\
\text { free survival }\end{array}$ & Reference \\
\hline Temsirolimus 250 mg/wk & 35 & $38 \%$ & $3 \%$ & $12 \mathrm{mo}$ & $6.5 \mathrm{mo}$ & Witzig et $\mathrm{al}^{76}$ \\
\hline Temsirolimus 25 mg/wk & 29 & $41 \%$ & $3.7 \%$ & $14 \mathrm{mo}$ & $6 \mathrm{mo}$ & Ansell et $\mathrm{al}^{78}$ \\
\hline Temsirolimus $175-75$ vs & 162 & $22 \%$ vs & & II.I mo vs & $7.4 \mathrm{mo}$ vs & Hess et $\mathrm{al}^{79}$ \\
\hline \multirow[t]{2}{*}{ I75-25 vs chosen therapy } & & $6 \%$ vs $2 \%$ & & $8.8 \mathrm{mo}$ vs & $3.4 \mathrm{mo} v \mathrm{~s}$ & \\
\hline & & & & $9.5 \mathrm{mo}$ & $1.6 \mathrm{mo}$ & \\
\hline $\begin{array}{l}\text { Temsirolimus } 25 \text { mg/wk + } \\
\text { rituximab } 4 \times\end{array}$ & 71 & $48 \%$ & $20 \%$ & & $9.5 \mathrm{mo}$ & Ansell et $\mathrm{al}^{81}$ \\
\hline
\end{tabular}


For some tumors, maximizing the bioavailability and dose intensity via intravenous administration may provide optimal clinical benefit. Temsirolimus is an ester analog of rapamycin that retains its potent intrinsic mTOR inhibitory activity while exhibiting better solubility for intravenous formulation. In the treatment of advanced renal cell carcinoma, temsirolimus is administered as a 30- to 60-minute infusion once weekly at a flat dose of $25 \mathrm{mg}$. This dosage results in high peak temsirolimus concentrations and limited immunosuppressive activity. ${ }^{75}$

From April 2002 to October 2003, 35 MCL patients (median age of 70 years, $91 \%$ in stage IV) who failed therapies with alkylating agents, cyclophosphamide, anthracyclines, purine analogs, and rituximab received $250 \mathrm{mg}$ of temsirolimus weekly for 6-12 cycles $^{76}$ (Table 2 ). The overall response rate was $38 \%$, with $3 \%$ of complete remissions and $35 \%$ of partial responses achieved by 3 months. The median time to progression was 6.5 months, median overall survival was 12 months, and the median duration of response was 6.9 months. Nevertheless, dose reduction was necessary in 31 cases: $71 \%$ of patients experienced grade 3 and 9\% experienced grade 4 hematological toxicity. Thrombocytopenia, anemia, neutropenia, increased triglycerides, diarrhea, hyperglycemia, sensory neuropathy, and rash were the most frequent adverse events.

The most frequent toxicities reported for mTOR inhibitors are mucositis, stomatitis, pneumonitis, rash, nail dystrophy, hyperlipidemia, hyperglycemia, and bone marrow suppression. In phase II trials in renal and breast cancer, the overall favorable safety profile of temsirolimus was confirmed; grade 3-4 mucositis was observed in $<5 \%$, skin rash in $4 \%$, pneumonitis in $5 \%$, hyperlipidemia in $6 \%$, hyperglycemia in $17 \%$, thrombocytopenia in $<5 \%$, and anemia in $9 \%$ of treated patients. ${ }^{77}$ Thrombocytopenia grade 3-4 occurred in $65 \%$, anemia in $25 \%$, and neutropenia in $28 \%$ of MCL cases; this difference was probably due to the frequent involvement of bone marrow ( $91 \%$ of patients were in stage IV) and treatments previously received. Nevertheless, only 1 patient required platelet transfusion and 4 required red cells support. The lower dose levels were evaluated in a phase II trial by the North Central Cancer Treatment Group. ${ }^{78}$

The objective of this study was to test a low dose of temsirolimus (25 mg weekly) in patients with relapsed MCL. Patients who had a tumor response after 6 cycles were eligible to continue the drug for a total of 12 cycles or 2 cycles after complete remission. The median age of the 29 enrolled patients was 69 years, with $86 \%$ of patients in stage IV. The overall response rate was $41 \%$, with $37 \%$ of partial responses. The median overall survival from the study entry was 14 months, and the time to progression and median duration of response were 6 months. Hematological toxicities were the most common toxicities, with $50 \%$ grade 3 and $4 \%$ grade 4 adverse events. Thrombocytopenia was the most frequent cause of dose reduction. Three patients experienced infection without concomitant neutropenia. Thus, this trial reported responses similar to those described for higher dose, but with less toxicity.

From June 2005 to July 2007, 162 patients were randomly assigned to receive temsirolimus at 2 doses ( $175 \mathrm{mg} / \mathrm{wk}$ for 3 weeks and then $75 \mathrm{mg}$ or $25 \mathrm{mg} /$ week) or a treatment chosen by the single investigator among the most frequently adopted agents for the treatment of relapsed MCL (gemcitabine, fludarabine, chlorambucil, cladribine, etoposide, thalidomide, vinblastine, alemtuzumab, lenalidomide). ${ }^{79}$ Median progression-free survival was $4.8,3.4$, and 1.9 months for temsirolimus $175 / 75 \mathrm{mg}$, temsirolimus $175 / 25 \mathrm{mg}$, and investigator's choice groups, respectively. Patients treated with temsirolimus $175 / 75 \mathrm{mg}$ had significantly longer progressionfree survival than those treated with investigator's choice therapy; those treated with temsirolimus 175/25 mg showed a trend toward longer progression-free survival. The objective response rate was significantly higher in the $175 / 75 \mathrm{mg}$ group (22\%) than in the investigator's choice group (2\%). The median overall survival for the temsirolimus $175 / 75 \mathrm{mg}$ group and the investigator's choice group was 12.8 and 9.7 months, respectively. The advantage offered by temsirolimus was independent of age, sex, performance status, stage of disease at diagnosis, number of extranodal sites, and blastoid histology. The most frequent grade 3 or 4 adverse events documented in $89 \%$ of patients in the temsirolimus $175 / 75 \mathrm{mg}$ group and in $80 \%$ of patients in the temsirolimus $175 / 25$ group were thrombocytopenia, anemia, neutropenia, and asthenia. Grade 3/4 thrombocytopenia was higher in the temsirolimus $175 / 75 \mathrm{mg}$ group (59\% vs $36 \%$ in the investigator's choice arm), anemia was comparable in the 2 arms (20\% vs $17 \%$ ), and neutropenia was lower in temsirolimus group (15\% vs $26 \%$ ). In the group of patients treated with temsirolimus, the most frequent side effects were asthenia (13\% vs $8 \%$ ), infection ( $9 \%$ vs $4 \%$ ), diarrhea (7\% vs $0 \%$ ), rash (7\% vs $0 \%$ ), dyspnea (7\% vs $9 \%$ ), fever ( $6 \%$ vs $0 \%$ ), and pruritus ( $4 \%$ vs $0 \%$ ). Nevertheless, a lower number of patients discontinued treatment with temsirolimus compared with those receiving standard therapies ( $43 \%$ vs 52\%), maintaining also a good quality of life.

At the 2009 European Hematology Association meeting, results from this trial were updated. The median progression-free survival and overall survival were confirmed to be significantly longer for the patients treated 
with $175 / 75 \mathrm{mg}$ temsirolimus than for those treated with investigator's choice therapy (5.2 vs 1.9 months and 5.2 vs 2 months, respectively). ${ }^{80}$

Moreover, considering the ability of rituximab to inhibit Akt and Raf-1 signalling pathways, 71 relapsed/resistant MCL patients received $25 \mathrm{mg}$ of intravenous temsirolimus every week and 4 weekly doses of rituximab in the first cycle, and then 1 dose of rituximab every other cycle, between May 2005 and March 2009. ${ }^{81}$ Patients with a tumor response after 6 cycles were eligible to continue treatment for a total of 12 cycles or 2 cycles after complete remission, and were then observed without maintenance. With median age of 67 years, 28\% of patients were rituximab refractory. The overall response rate was $48 \%$, with $20 \%$ of complete and $28 \%$ of partial responses. The median duration of response was 9.5 months for rituximabsensitive patients and 7.15 months for rituximab-refractory patients. While the combination was generally well tolerated, 12 patients experienced grade 4 toxicity. Hematological toxicities were most common, with 5 patients having grade 4 thrombocytopenia and 3 having grade 4 neutropenia.

Responses (30\%) to temsirolimus by patients with MCL are higher than those reported by patients with renal cell carcinoma, breast cancer, lung cancer, and glioblastoma (about 10\%). Why patients affected by lymphoma would be more sensitive than those affected by solid tumors is still unexplained; some authors suggest that the different ways of inactivation of PTEN (phosphorylation in lymphoma and mutation/deletion in solid tumors) could be one possible cause. It is also possible that prolonged exposure to temsirolimus could inactivate both the mTORC1 and mTORC2 complexes in sensitive tumors, therefore inhibiting the negative feedback of phosphorylation of Akt. ${ }^{53}$

Considering these promising results, the future perspective would be to test other mTOR inhibitors, such as everolimus, or drug combinations in MCL, but as first-line therapies.As reported in the 2009 American Society of Hematology meeting, 37 patients with relapsed diffuse large cell lymphoma and MCL received $5 \mathrm{mg}$ /day of everolimus. The overall response rate was $32 \%$, with $29 \%$ in the MCL group. The median time to progression for all patients was 3.1 months. The median duration of response for the 12 responders was 5.5 months. Everolimus was well tolerated, with the incidence of grade 3 anemia, neutropenia, and thrombocytopenia occurring in $11 \%, 16 \%$, and $30 \%$ of cases. ${ }^{82}$

Thus, the perspective of using mTOR inhibitors as first-line treatment for MCL patients would be a challenge for the next future; the association of rituximab, cladribine, and temsirolimus is scheduled for the newly diagnosed MCL (registered at
ClinicalTrials.gov as NCT00787969, April 2009). This phase I/II trial is planned to evaluate the efficacy and safety of temsirolimus when given together with cladribine and rituximab; the treatment is repeated every 28 days for up to 6 courses in the absence of disease progression or unacceptable toxicity. As secondary endpoints, the trial includes the assessment of metabolic markers (hyperglycemia and hyperlipidemia) as markers of mTOR inhibition and correlation of response with serum-free light chains, single-nucleotide polymorphisms in host immune genes, vitamin D metabolites, and PI3K pathway member expression.

It is probable that patients not heavily pretreated could represent the best scenario for using mTOR inhibitors to reduce toxicities and increase their efficacies.

In conclusion, the demonstration that temsirolimus at $175 \mathrm{mg} /$ week as induction, followed by $75 \mathrm{mg} /$ week, significantly improved clinical benefit (response rate and progressionfree survival) in comparison to "canonical" drugs is the most convincing element for considering the use of this mTOR inhibitor in relapsed MCL patients. Moreover, the fact that temsirolimus is well tolerated in general and that severe clinical side effects $>$ grade 3 are rare events represents another supporting element. Obviously, the optimal dose of temsirolimus, as well as the exploration of new combinations (in particular with anti-CD20 antibodies, histone deacetylase inhibitors and inhibitors of Raf or angiogenesis), is still under discussion, and further studies enrolling larger series of patients are warranted to confirm the above reported promising clinical results.

\section{Disclosure}

The authors report no conflicts of interest in this work.

\section{References}

1. Zucca E, Roggero E, Pinotti G, et al. Patterns of survival in mantle cell lymphoma. Ann Oncol. 1995;6(3):257-262.

2. Romaguera JE, Fayad L, Rodriguez MA, et al. High rate of durable remissions after treatment of newly diagnosed aggressive mantlecell lymphoma with rituximab plus hyper-CVAD alternating with rituximab plus high-dose methotrexate and cytarabine. J Clin Oncol. 2005;23(28):7013-7023.

3. Lenz G, Dreyling M, Hoster E, et al. Immunochemotherapy with rituximab and cyclophosphamide, doxorubicin, vincristine, and prednisone significantly improves response and time to treatment failure, but not long-term outcome in patients with previously untreated mantle cell lymphoma: results of a prospective randomized trial of the German Low Grade Lymphoma Study Group (GLSG). J Clin Oncol. 2005;23(9):1984-1992.

4. Forstpointner R, Dreyling M, Repp R, et al. The addition of rituximab to a combination of fludarabine, cyclophosphamide, mitoxantrone (FCM) significantly increases the response rate and prolongs survival as compared with FCM alone in patients with relapsed and refractory follicular and mantle cell lymphomas: results of a prospective randomized study of the German Low-Grade Lymphoma Study Group. Blood. 2004;104(10):3064-3071. 
5. Wang M, Oki Y, Pro B, et al. Phase II study of yttrium-90-ibritumomab tiuxetan in patients with relapsed or refractory mantle cell lymphoma. J Clin Oncol. 2009;27(31):5213-5218.

6. O'Connor OA, Moskowitz C, Portlock C, et al. Patients with chemotherapy-refractory mantle cell lymphoma experience high response rates and identical progression-free survivals compared with patients with relapsed disease following treatment with single agent bortezomib: results of a multicentre Phase 2 clinical trial. Br J Haematol. 2009;145(1):34-39.

7. Habermann TM, Lossos IS, Justice G, et al. Lenalidomide oral monotherapy produces a high response rate in patients with relapsed or refractory mantle cell lymphoma. Br J Haematol. 2009;145(3): 344-349.

8. Geisler C. Mantle cell lymphoma: are current therapies changing the course of disease? Curr Oncol Rep. 2009;11(5):371-377.

9. Sehgal SN, Baker H, Vézina C. Rapamycin (AY-22,989), a new antifungal antibiotic. II. Fermentation, isolation and characterization. J Antibiot (Tokyo). 1975;28(10):727-732.

10. Drakos E, Rassidakis GZ, Medeiros LJ. Mammalian target of rapamycin (mTOR) pathway signalling in lymphomas. Expert Rev Mol Med. 2008; 10:e4.

11. Gaumann A, Schlitt HJ, Geissler EK. Immunosuppression and tumor development in organ transplant recipients: the emerging dualistic role of rapamycin. Transpl Int. 2008;21(3):207-217.

12. Bjornsti MA, Houghton PJ. The TOR pathway: a target for cancer therapy. Nat Rev Cancer. 2004;4(5):335-348.

13. Gibbons JJ, Abraham RT, Yu K. Mammalian target of rapamycin: discovery of rapamycin reveals a signaling pathway important for normal and cancer cell growth. Semin Oncol. 2009;36 Suppl 3:S3-S17.

14. Cafferkey R, McLaughlin MM, Young PR, Johnson RK, Livi GP. Yeast TOR (DRR) proteins: amino-acid sequence alignment and identification of structural motifs. Gene. 1994;141(1):133-136.

15. Hay N, Sonenberg N. Upstream and downstream of mTOR. Genes Dev. 2004;18(16):1926-1945.

16. Jozwiak J, Jozwiak S, Grzela T, Lazarczyk M. Positive and negative regulation of TSC2 activity and its effects on downstream effectors of the mTOR pathway. Neuromolecular Med. 2005;7(4):287-296.

17. Potter CJ, Pedraza LG, Xu T. Akt regulates growth by directly phosphorylating Tsc2. Nat Cell Biol. 2002;4(9):658-665.

18. Wullschleger S, Loewith R, Hall MN. TOR signaling in growth and metabolism. Cell. 2006;124(3):471-484.

19. Vignot S, Faivre S, Aguirre D, Raymond E. mTOR-targeted therapy of cancer with rapamycin derivatives. Ann Oncol. 2005;16(4):525-537.

20. Wullschleger S, Loewith R, Oppliger W, Hall MN. Molecular organization of target of rapamycin complex 2. J Biol Chem. 2005; 280(35):30697-30704.

21. Julien LA, Carriere A, Moreau J, Roux PP. mTORC1-activated S6K1 phosphorylates Rictor on threonine 1135 and regulates mTORC2 signaling. Mol Cell Biol. 2010;30(4):908-921.

22. Gingras AC, Gygi SP, Raught B, et al. Regulation of 4E-BP1 phosphorylation: a novel two-step mechanism. Genes Dev. 1999;13(11): 1422-1437.

23. Mirshahi P, Toprak SK, Faussat AM, et al. Malignant hematopoietic cells induce an increased expression of VEGFR-1 and VEGFR-3 on bone marrow endothelial cells via AKT and mTOR signalling pathways. Biochem Biophys Res Commun. 2006;349(3):1003-1010.

24. Baldo P, Cecco S, Giacomin E, Lazzarini R, Ros B, Marastoni S. mTOR pathway and mTOR inhibitors as agents for cancer therapy. Curr Cancer Drug Targets. 2008;8(8):647-665.

25. Brown VI, Seif AE, Reid GS, Teachey DT, Grupp SA. Novel molecular and cellular therapeutic targets in acute lymphoblastic leukemia and lymphoproliferative disease. Immunol Res. 2008;42(1-3):84-105.

26. Avellino R, Romano S, Parasole R, et al. Rapamycin stimulates apoptosis of childhood acute lymphoblastic leukemia cells. Blood. 2005; 106(4):1400-1406.

27. Crazzolara R, Cisterne A, Thien M, et al. Potentiating effects of RAD001 (Everolimus) on vincristine therapy in childhood acute lymphoblastic leukemia. Blood. 2009;113(14):3297-3306.
28. Gu L, Gao J, Li Q, et al. Rapamycin reverses NPM-ALK-induced glucocorticoid resistance in lymphoid tumor cells by inhibiting mTOR signaling pathway, enhancing G1 cell cycle arrest and apoptosis. Leukemia. 2008;22(11):2091-2096.

29. Kharas MG, Deane JA, Wong S, et al. Phosphoinositide 3-kinase signaling is essential for ABL oncogene-mediated transformation of B-lineage cells. Blood. 2004;103(11):4268-4275.

30. Xu Q, Simpson SE, Scialla TJ, Bagg A, Carroll M. Survival of acute myeloid leukemia cells requires PI3 kinase activation. Blood. 2003; 102(3):972-980.

31. Follo MY, Mongiorgi S, Bosi C, et al. The Akt/mammalian target of rapamycin signal transduction pathway is activated in high-risk myelodysplastic syndromes and influences cell survival and proliferation. Cancer Res. 2007;67(9):4287-4294.

32. Hoang B, Benavides A, Shi Y, Frost P, Lichtenstein A. Effect of autophagy on multiple myeloma cell viability. Mol Cancer Ther. 2009; 8(7):1974-1984.

33. Frost $\mathrm{P}$, Shi $\mathrm{Y}$, Hoang B, Lichtenstein A. AKT activity regulates the ability of mTOR inhibitors to prevent angiogenesis and VEGF expression in multiple myeloma cells. Oncogene. 2007;26(16):2255-2262.

34. Farag SS, Zhang S, Jansak BS, et al. Phase II trial of temsirolimus in patients with relapsed or refractory multiple myeloma. Leuk Res. 2009;33(11):1475-1480.

35. Dutton A, Reynolds GM, Dawson CW, Young LS, Murray PG. Constitutive activation of phosphatidyl-inositide 3 kinase contributes to the survival of Hodgkin's lymphoma cells through a mechanism involving Akt kinase and mTOR. J Pathol. 2005;205(4):498-506.

36. Gupta M, Ansell SM, Novak AJ, Kumar S, Kaufmann SH, Witzig TE. Inhibition of histone deacetylase overcomes rapamycin-mediated resistance in diffuse large B-cell lymphoma by inhibiting Akt signaling through mTORC2. Blood. 2009;114(14):2926-2935.

37. Pop IV, Pop LM, Ghetie MA, Vitetta ES. Targeting mammalian target of rapamycin to both downregulate and disable the P-glycoprotein pump in multidrug-resistant B-cell lymphoma cell lines. Leuk Lymphoma. 2009;50(7):1155-1162.

38. Gasperini P, Tosato G. Targeting the mammalian target of Rapamycin to inhibit VEGF and cytokines for the treatment of primary effusion lymphoma. Leukemia. 2009;23(10):1867-1874.

39. Nepomuceno RR, Balatoni CE, Natkunam Y, Snow AL, Krams SM, Martinez OM. Rapamycin inhibits the interleukin 10 signal transduction pathway and the growth of Epstein Barr virus B-cell lymphomas. Cancer Res. 2003;63(15):4472-4480.

40. Leseux L, Hamdi SM, Al Saati T, et al. Syk-dependent mTOR activation in follicular lymphoma cells. Blood. 2006;108(13):4156-4162.

41. Gupta M, Dillon SR, Ziesmer SC, et al. A proliferation-inducing ligand mediates follicular lymphoma B-cell proliferation and cyclin D1 expression through phosphatidylinositol 3-kinase-regulated mammalian target of rapamycin activation. Blood. 2009;113(21): 5206-5216.

42. Ringshausen I, Peschel C, Decker T. Mammalian target of rapamycin (mTOR) inhibition in chronic lymphocytic B-cell leukemia: a new therapeutic option. Leuk Lymphoma. 2005;46(1):11-19.

43. Decker T, Hipp S, Ringshausen I, et al. Rapamycin-induced G1 arrest in cycling B-CLL cells is associated with reduced expression of cyclin D3, cyclin E, cyclin A, and survivin. Blood. 2003;101(1):278-285.

44. Decker T, Sandherr M, Goetze K, Oelsner M, Ringshausen I, Peschel C. A pilot trial of the mTOR (mammalian target of rapamycin) inhibitor RAD001 in patients with advanced B-CLL. Ann Hematol. 2009;88(3):221-227.

45. Jares P, Colomer D, Campo E. Genetic and molecular pathogenesis of mantle cell lymphoma: perspectives for new targeted therapeutics. Nat Rev Cancer. 2007;7(10):750-762.

46. Decaudin D. Mantle cell lymphoma: a biological and therapeutic paradigm. Leuk Lymphoma. 2002;43(4):773-781.

47. Sherr CJ. D-type cyclins. Trends Biochem Sci. 1995;20(5):187-190.

48. Dal Col J, Zancai P, Terrin L, et al. Distinct functional significance of Akt and mTOR constitutive activation in mantle cell lymphoma. Blood. 2008;111(10):5142-5151. 
49. Rudelius M, Pittaluga S, Nishizuka S, et al. Constitutive activation of Akt contributes to the pathogenesis and survival of mantle cell lymphoma. Blood. 2006;108(5):1668-1676.

50. Haritunians T, Mori A, O’Kelly J, Luong QT, Giles FJ, Koeffler HP. Antiproliferative activity of RAD001 (everolimus) as a single agent and combined with other agents in mantle cell lymphoma. Leukemia. 2007; 21(2):333-339.

51. Leseux L, Laurent G, Laurent C, et al. PKC zeta mTOR pathway: a new target for rituximab therapy in follicular lymphoma. Blood. 2008; 111(1):285-291.

52. Younes A. Therapeutic activity of mTOR inhibitors in mantle cell lymphoma. Autophagy 2008;4(5):707-709.

53. Peponi E. Activation of mammalian target of rapamycin signaling promotes cell cycle progression and protects cells from apoptosis in mantle cell lymphoma. Am J Pathol. 2006;169(6):2171-2180.

54. Hipp S, Ringshausen I, Oelsner M, Bogner C, Peschel C, Decker T. Inhibition of the mammalian target of rapamycin and the induction of cell cycle arrest in mantle cell lymphoma cells. Haematologica. 2005; 90(10):1433-1434.

55. Wolowiec D, Berger F, Ffrench P, Bryon PA, Ffrench M. CDK1 and cyclin A expression is linked to cell proliferation and associated with prognosis in non-Hodgkin's lymphomas. Leuk Lymphoma. 1999;35(1-2):147-157.

56. Gelebart P, Anand M, Armanious H, et al. Constitutive activation of the Wnt canonical pathway in mantle cell lymphoma. Blood. 2008; 112(13):5171-5179.

57. Yazbeck VY, Buglio D, Georgakis GV, et al. Temsirolimus downregulates p21 without altering cyclin D1 expression and induces autophagy and synergizes with vorinostat in mantle cell lymphoma. Exp Hematol. 2008;36(4):443-450.

58. Giatromanolaki A, Koukourakis MI, Pezzella F, et al. Phosphorylated VEGFR2/KDR receptors are widely expressed in B-cell non-Hodgkin's lymphomas and correlate with hypoxia inducible factor activation. Hematol Oncol. 2008;26(4):219-224.

59. Koomagi R, Zintl F, Sauerbrey A, Volm M. Vascular endothelial growth factor in newly diagnosed and recurrent childhood acute lymphoblastic leukemia as measured by real-time quantitative polymerase chain reaction. Clin Cancer Res. 2001;7(11):3381-3384.

60. Hazar B, Paydas S, Zorludemir S, Sahin B, Tuncer I. Prognostic significance of microvessel density and vascular endothelial growth factor (VEGF) expression in non-Hodgkin's lymphoma. Leuk Lymphoma. 2003;44(12):2089-2093.

61. Kuramoto K, Sakai A, Shigemasa K, et al. High expression of MCL1 gene related to vascular endothelial growth factor is associated with poor outcome in non-Hodgkin's lymphoma. Br J Haematol. 2002;116 (1): $158-161$

62. Potti A, Ganti AK, Kargas S, Koch M. Immunohistochemical detection of C-kit (CD117) and vascular endothelial growth factor (VEGF) overexpression in mantle cell lymphoma. Anticancer Res. 2002;22(5):2899-2901.

63. Galimberti S, Nagy B, Palumbo GA, et al. Vascular endothelial growth factor polymorphisms in mantle cell lymphoma. Acta Haematol. 2010; 123(2):91-95.

64. Roux PP, Shahbazian D, Vu H, et al. RAS/ERK signaling promotes sitespecific ribosomal protein S6 phosphorylation via RSK and stimulates cap-dependent translation. J Biol Chem. 2007;282(19):14056-14064.

65. Kurie JM, Morse HC III, Principato MA, et al. v-myc and v-raf act synergistically to induce B-cell tumors in pristane-primed adult BALBC mice. Oncogene. 1990;5(4):577-582.

\section{Cancer Management and Research}

\section{Publish your work in this journal}

Cancer Management and Research is an international, peer-reviewed open access journal focusing on cancer research and the optimal use of preventative and integrated treatment interventions to achieve improved outcomes, enhanced survival and quality of life for the cancer patient The journal welcomes original research, clinical \& epidemiological
66. Nagy B, Galimberti S, Benedetti E, et al. RAF-1 over-expression does condition survival of patients affected by aggressive mantle cell lymphoma. Leuk Res. 2007;31(11):1595-1597.

67. Nagy B, Lundán T, Larramendy ML, et al. Abnormal expression of apoptosis-related genes in hematological malignancies: overexpression of MYC is poor prognostic sign in mantle cell lymphoma. $\mathrm{Br} J$ Haematol. 2003;120(3):4434-4441.

68. Mills JR, Hippo Y, Robert F, et al. mTORC1 promotes survival through translational control of Mcl-1. Proc Natl Acad Sci USA. 2008; 105(31): 10853-10858.

69. Rinaldi A, Kwee I, Taborelli M, et al. Genomic and expression profiling identifies the B-cell associated tyrosine kinase Syk as a possible therapeutic target in mantle cell lymphoma. Br J Haematol. 2006;132(3):303-316.

70. Hudes GR, Berkenblit A, Feingold J, Atkins MB, Rini BI, Dutcher J. Clinical trial experience with temsirolimus in patients with advanced renal cell carcinoma. Semin Oncol. 2009;36 Suppl 3:S26-S36.

71. Mita MM, Mita A, Rowinsky EK. Mammalian target of rapamycin: a new molecular target for breast cancer. Clin Breast Cancer. 2003;4(2):126-137.

72. Mita MM, Tolcher AW. The role of mTOR inhibitors for treatment of sarcomas. Curr Oncol Rep. 2007;9(4):316-322.

73. Tolcher AW. Novel therapeutic molecular targets for prostate cancer: the mTOR signaling pathway and epidermal growth factor receptor. $J$ Urol. 2004;171:S41-S43; discussion S44.

74. Coiffier B, Ribrag V. Exploring mammalian target of rapamycin (mTOR) inhibition for treatment of mantle cell lymphoma and other hematologic malignancies. Leuk Lymphoma. 2009;50(12):1916-1930.

75. Boni JP, Hug B, Leister C, Sonnichsen D. Intravenous temsirolimus in cancer patients: clinical pharmacology and dosing considerations. Semin Oncol. 2009;36 Suppl 3:S18-S25.

76. Witzig TE, Geyer SM, Ghobrial I, et al. Phase II trial of single-agent temsirolimus (CCI-779) for relapsed mantle cell lymphoma. J Clin Oncol. 2005;23(23):5347-5356.

77. Sankhala K, Mita A, Kelly K, Mahalingam D, Giles F, Mita M. The emerging safety profile of mTOR inhibitors, a novel class of anticancer agents. Target Oncol. 2009;4(2):135-142.

78. Ansell SM, Inwards DJ, Rowland KM Jr, et al. Low-dose, single-agent temsirolimus for relapsed mantle cell lymphoma: a phase 2 trial in the North Central Cancer Treatment Group. Cancer. 2008;113(3):508-514.

79. Hess G, Herbrecht R, Romaguera J, et al. Phase III study to evaluate temsirolimus compared with investigator's choice therapy for the treatment of relapsed or refractory mantle cell lymphoma. J Clin Oncol. 2009;27(23):3822-3829.

80. Hess G, Romaguera J, Herbrecht R, et al. Temsirolimus for the treatment of patients with relapsed or refractory mantle cell lymphoma. Supportive efficacy analyses from the phase 3 study. Haematologica. 2009;94 Supp1 2:S391. Abstract 0973.

81. Ansell SM, Tang H, Kurtin P, et al. A phase II study of temsirolimus (CCI-779) in combination with rituximab in patients with relapsed or refractory mantle cell lymphoma [ASH Annual Meeting Abstracts]. Blood. 2009;114:1665.

82. Reeder CB, Gornet MK, Habermann TM, et al. A phase II trial of the oral mTOR inhibitor everolimus (RAD001) in relapsed aggressive nonHodgkin lymphoma (NHL) [ASH Annual Meeting Abstracts]. Blood. 2007;110:121.

studies, reviews \& evaluations, guidelines, expert opinion \& commentary, case reports \& extended reports. The manuscript management system is completely online and includes a very quick and fair peerreview system, which is all easy to use. Visit http://www.dovepress.com/ testimonials.php to read real quotes from published authors. 